\title{
A GENERALIZED CLASS OF TRANSFORMATION MATRICES FOR THE RECONSTRUCTION OF SPHERE SIZE DISTRIBUTIONS FROM SECTION CIRCLE SIZE DISTRIBUTIONS
}

\author{
"WILLI PABST, TEREZA UHLÍŘOVÁ \\ Department of Glass and Ceramics, University of Chemistry and Technology, Prague \\ Technická 5, 16628 Prague 6, Czech Republic \\ \#E-mail: willi.pabst@vscht.cz
}

Submitted November 25, 2016; accepted January 28, 2017

\begin{abstract}
Keywords: Random section problem, Wicksell's corpuscle problem, Saltykov transformation, Cruz-Orive transformation, Wicksell transformation, Woodhead transformation, spherical inclusions (pores, grains, particles), planar / polished sections, microscopic image analysis, sphere / section circle diameter, monosized spheres, monodisperse / monomodal distribution, Dirac distribution, bidisperse / bimodal distribution, uniform (rectangular) distribution, linear (triangular saw-tooth) distribution, Rayleigh distribution, frequency / cumulative histogram, arithmetic mean size

A generalized formulation of transformation matrices is given for the reconstruction of sphere diameter distributions from their section circle diameter distributions. This generalized formulation is based on a weight shift parameter that can be adjusted from 0 to 1. It includes the well-known Saltykov and Cruz-Orive transformations as special cases (for parameter values of 0 and 0.5 , respectively). The physical meaning of this generalization is explained (showing, among others, that the Woodhead transformation should be bounded by the Saltykov transformation on the one side and by our transformation from the other) and its numerical performance is investigated. In particular, it is shown that our generalized transformation is numerically highly unstable, i.e. introduces numerical artefacts (oscillations or even unphysical negative sphere frequencies) into the reconstruction, and can lead to completely wrong results when a critical value of the parameter (usually in the range $0.7-0.9$, depending on the type of distribution) is exceeded. It is shown that this numerical instability is an intrinsic feature of these transformations that depends not only on the weight shift parameter value and is affected both by the type and the position of the distribution. It occurs in a natural way also for the Cruz-Orive and other transformations with finite weight shift parameter values and is not just caused by inadequate input data (e.g. as a consequence of an insufficient number of objects counted), as commonly assumed. Finally it is shown that an even more general class of transformation matrices can be defined that includes, in addition to the aformentioned transformations, also the Wicksell transformation.
\end{abstract}

\section{INTRODUCTION}

When simply connected objects are embedded in a microstructure, their size distribution can be defined and, in principle, determined. However, only in exceptional cases spatial (tomographic) images, e.g. in the form of discretized voxel models, are available from which the size distribution can be determined directly. Much more common in materials science is the situation that only planar sections are available. The question how to determine the diameter distribution of spherical and spheroidal inclusions or pores embedded in a threedimensional (3D) microstructure from the section circle diameter distribution on a two-dimensional (2D) planar cut through this microstructure has been for the first time correctly posed and answered by Wicksell in 1925 and 1926 [1, 2]. Wicksell studied convex objects in biological tissues, but was fully aware of the implications of his results for other fields of science, including materials science and astronomy. Indeed, Wicksell's random section problem for spherical or spheroidal inclusions in microstructures $[1,2]$, sometimes called "Wicksell's corpuscle problem" (due to the biometric context in which Wicksell formulated it for the first time), is one of the classical problems of stereology. From the physical point of view the problem consists in reconstructing the $3 \mathrm{D}$ sphere diameter distribution from the $2 \mathrm{D}$ section circle diameter distribution, while from the mathematical point of view this so-called inverse problem consists in the approximate solution of an Abeltype integral equation (or a Volterra integral equation of the first kind) [3, 4]. In his first paper on that topic [1] Wicksell proposed a correct approximate solution that could be applied to arbitrary frequency histograms and that could be expressed in the form of a simple universal transformation matrix. The practical application of his transformation matrix, however, was impeded by the fact that he used a rather awkward representation of his histograms by relating the individual columns or size classes (bins) to the interval midpoints, which required an extra treatment for the half-interval from zero to the lower limit of the first bin. In the sequel, Scheil [5], Schwartz [6] and Saltykov [7] reconsidered Wicksell's random section problem for spheres and, based on Wicksell's work, proposed an alternative transformation matrix applicable to histograms with full bins only, 
starting from zero. This representation is common standard today. Saltykov's work, on the other hand, was published first in Russian (in the second edition of his book "Stereometric Metallography" from 1958 [7]), and did not become available in a Western language until 1974 in the form of a German translation [8] of the third Russian edition [9] (a Czech translation of the second Russian edition [7] was published in 1968 [10]). This is the reason why Cruz-Orive in his 1976 paper [11] was obviously not aware of Saltykov's work. In that paper and follow-up paper published in 1978 [12] Cruz-Orive developed another approximate solution of Wicksell's random section problem for spheroids and derived a different transformation matrix for spheres, again for the aforementioned standard histograms (on which also Saltykov's matrix is based). However, in contrast to Wicksell and Saltykov, Cruz-Orive suggested using the interval midpoints (and not their upper limits) of the sphere size classes for statistical weighting (see below). Independently, the result for spheres had been obtained before by Goldsmith in 1967 [13], but CruzOrive, whose primary aim was to calculate size-shape distributions of spheroids (after having proved that the general triaxial ellipsoid problem is not only unsolvable but even indeterminate), was obviously not aware of that earlier work on spheres. Still another transformation matrix for spheres has been developed by Woodhead in 1980 or before. It seems that this matrix has never been published by Woodhead himself, but it is cited as a "private communication" in a more recent (2003) book by Higginson and Sellars [14] and corresponds to the transformation matrix derived (probably independently from Woodhead) by Blödner et al. in an appendix to their 1984 paper [15], assuming a "uniform distribution" within each sphere size class for the statistical weighting (instead of weighting the size classes by the interval midpoints, as Cruz-Orive did, or by the interval upper limits, as Saltykov did). All these transformations (called Saltykov, Cruz-Orive and Woodhead in the sequel) are based on the aforementioned standard representation of histograms and have been obtained as approximate solutions of the aforementioned inverse problem (Abel-type integral equation). Early discussions of this approach have been given by Underwood [16] and Lewis et al. [17]. Very recently all three transformations have been successfully used for reconstructing Rayleighdistributed grain size distributions in fully sintered ceramics [18]. Numerical aspects of the solution of Abel-type integral equations have been discussed by Anderssen and Jakeman in 1975 [19, 20], and a recent $\mathrm{PhD}$ thesis [21] proves that the numerical aspect of solutions to Wicksell's random section problem is still a topic of immense interest. It has been reported in the literature several times $[15,22,23]$, that the Cruz-Orive and Woodhead transformations lead to very similar results and that they are usually not "better" than the Saltykov transformation. On the contrary, they seem to be more prone to exhibit numerical instabilities which produce artefacts and may lead to errors (e.g. negative frequencies) in the reconstructed sphere size distributions. This problem will be more thoroughly investigated in a follow-up paper [24]. However, recalling all the aforesaid, it might be surprising why authors always used a statistical weighting using upper limit values, midpoints or assuming uniform distributions inside each size class and never considered the use of lower limit values for this purpose. Indeed this preference is not without reason, but this reason has never been explicitly stated, and it is well thinkable that the lower limit values are more representative of a size class, e.g. for the decreasing branch of a distribution. Therefore, in the present paper we propose a generalized formulation of the transformation matrix for reconstructing the sphere diameter distribution from the section circle diameter distribution. This generalized version contains the Saltykov and Cruz-Orive transformation matrices as special cases and should provide a bound also for the Woodhead transformation. By adjusting a weight parameter it can be fine-tuned in order to provide the most realistic representation of the size distribution to be expected, this giving room for further developments. It is, however, prone to numerical instability when the size class is weighted by a value too close to the lower limit. Based on a few paradigmatic examples, the critical values are indicated and it is shown how the numerical instability of this generalized transformation explains in a natural way the artefacts occurring in the CruzOrive transformation. Albeit unusual, our formulation can be readily extended to include even the Wicksell transformation. Also this is shown.

\section{THEORETICAL BACKGROUND AND PRACTICAL CONSEQUENCES}

The section circle diameter distribution is usually different from the sphere diameter distribution because of two reasons: First, the section circle diameters are almost always smaller than the sphere diameters; although the size class with largest section circles is the most frequent one, section circles with a diameter corresponding exactly to the sphere diameter occur with a probability of zero. Second, the probability of cutting isotropically, uniformly and randomly arranged spheres embedded in a microstructure by a random section plane is higher for large spheres than for small spheres; in particular, setting the probability of cutting a sphere of diameter $D$ to 1 (unity), the probability of cutting spheres of diameters $2 D, 3 D, \ldots \mathrm{j} D$ by a random section plane is $1,2,3, \ldots j$, respectively. In his first memoir, Wicksell [1] pointed out that these two effects are counteracting and may partly or completely counterbalance each other. That means, in contrast to widespread belief, there is no reason to assume that the section circle diameter distribution is necessarily 
shifted to smaller sizes compared to the sphere diameter distribution. And indeed there is a case where the two effects exactly counterbalance each other: the Rayleigh distribution. The frequency curve (probability density) of this one-parameter distribution is defined as [4]

$$
p(x)=\frac{x}{a^{2}} \exp \left(-\frac{x^{2}}{2 a^{2}}\right),
$$

where $x$ is either the sphere diameter $D$ or the section circle diameter $d$, and the Rayleigh parameter $a$ determines the distribution and all its characteristic values completely, e.g. the arithmetic mean $\bar{x}=a \cdot \sqrt{\pi / 2}$. It has been used e.g. by Wicksell [1], Rysz and Wiencek [25] and Blödner et al. [15] to evaluate and compare the performance of reconstruction procedures. On the other hand, Drees and Reiss [26] have proved that the Rayleigh distribution is the only distribution with this property. Therefore it is justified to conclude reversely that if the transformation procedure leaves the section circle diameter distribution unchanged (or almost unchanged), the section circle diameter distribution is equal (or almost equal) to the original sphere diameter distribution and that this sphere diameter distribution equals (at least approximately) a Rayleigh distribution [18].

The practical application of transformation matrices is very simple, since frequency histograms of size classes, e.g. section circle or sphere diameters, can be considered as abstract $i$ - or $j$-dimensional vectors, denoted as $n_{i}$ and $N_{j}$, respectively. In fact, $n_{i}$ and $N_{j}$ may also be considered as the number of section circles per unit area of the planar section and the number of spheres per unit volume in the respective size classes, in stereological standard notation denoted as $N_{A i}$ and $N_{V j}$, respectively. When the size classes of spheres and section circles are chosen to be the same (which is common practice) the vector $n_{i}$ is related to the vector $N_{j}$ a matrix transformation of the type

$$
n_{i}=\Delta \cdot B_{i j} N_{j}=\Delta \cdot \sum_{j} B_{i j} N_{j},
$$

where $\Delta$ is the interval width (which can be omitted when only relative frequencies, i.e. normalized frequency histograms are considered), the Einstein summation convention has been used (summation over adjacent repeated indices) and the summation symbol has been omitted. When the sphere diameter distribution, i.e. the vector $N_{j}$, is known (e.g. for a chosen model distribution in the form of a histogram), this relation can be used to calculate the vector $n_{i}$, i.e. to construct the corresponding section circle distribution using the "section circle construction matrix"

$$
B_{i j}=\sqrt{j^{2}-(i-1)^{2}}-\sqrt{j^{2}-i^{2}}
$$

for $i \leq j$ and $B_{i j}=0$ for $i>j$ (expressing the fact that section circles cannot be larger than the spheres which are cut). It should be emphasized that Equation 3 is the only correct "section circle construction matrix". It is derived by a procedure that is completely analogous to the procedure used by Saltykov [7-10]. When considered in greater detail it can be seen that Equation 3 is a product of two terms, one that allows the calculation of the probability density of section circle diameters $d_{i}$ and $d_{i-1}$ for a given sphere diameter $D_{j}[3]$,

$$
P_{i j}=\frac{\sqrt{D_{j}^{2}-d_{i-1}^{2}}-\sqrt{D_{j}^{2}-d_{i}^{2}}}{D_{j}}
$$

and one that takes into account the fact that larger spheres have a higher probability to be cut at all. The latter effect is taken into account by a statistical weight factor for each sphere diameter. When section circles are to be obtained it is plausible that this weight factor is just the sphere diameter $D_{j}$ itself, because for spheres with a diameter of $2 D_{j}$ the probability to be cut by a random section plane is twice as high as for spheres with a diameter of $D_{j}$. It is clear, however, that the weight factor can also be any multiple of the sphere diameter $D_{j}$. In the section circle construction matrix these factors occur as the sums of the individual columns. For example, the sums of columns of the $(4 \times 4)$ section circle construction matrix calculated according to Equation 3, i.e.

$$
B_{i j}=\left(\begin{array}{cccc}
1 & 0.267949 & 0.171573 & 0.127017 \\
0 & 1.732051 & 0.592359 & 0.408882 \\
0 & 0 & 2.236068 & 0.81835 \\
0 & 0 & 0 & 2.645751
\end{array}\right)
$$

are 1, 2, 3 and 4, respectively. The inverse of the section circle construction matrix calculated according to Equation 3, i.e. the matrix $C_{i j}=B_{i j}{ }^{-1}$, can be used to reconstruct the (initially unknown) sphere diameter distribution from the (directly measured) section circle diameter distribution according to the relation

$$
N_{j}=\frac{1}{\Delta} \cdot n_{i} C_{i j}=\frac{1}{\Delta} \cdot \sum_{i} n_{i} C_{i j} .
$$

When the section circle construction matrix $B_{i j}$, i.e. Equation 3, is used to calculate the inverse matrix $C_{i j}$, this is what is usually called the Saltykov transformation for the reconstruction of sphere diameter distributions. For example, the $(4 \times 4)$ matrix

$$
C_{i j}=B_{i j}^{-1}=\left(\begin{array}{cccc}
1 & -0.1547 & -0.03575 & -0.01304 \\
0 & 0.57735 & -0.15295 & -0.04192 \\
0 & 0 & 0.447214 & -0.13833 \\
0 & 0 & 0 & 0.377964
\end{array}\right)
$$

is the Saltykov matrix for reconstructing a sphere diameter distribution by transforming a section circle histogram with 4 equally wide size classes, starting from zero, see [7-10].

The use of the Saltykov transformation amounts to weighting the size classes by their upper limit values. It is clear that this is not the only plausible way how this weighting can be done. Actually, from a physical point of view, when the sphere diameter distribution is 
unknown, it would seem much more realistic in many cases to refer to the midpoints of the sphere size classes for weighting, as has been suggested by Cruz-Orive $[11,12]$. In this case (Cruz-Orive transformation) the matrix for the reconstruction of sphere diameter distributions is the inverse of the matrix

$B_{i j}=\sqrt{\left(j-\frac{1}{2}\right)^{2}-(i-1)^{2}}-\sqrt{\left(j-\frac{1}{2}\right)^{2}-i^{2}}$ for $i<j$,

$B_{i j}=\sqrt{\left(j-\frac{1}{2}\right)^{2}-(i-1)^{2}}=B_{i i}=$

$=\sqrt{\left(i-\frac{1}{2}\right)^{2}-(i-1)^{2}}=\sqrt{i-\frac{3}{4}}$ for $i=j$,

and $B_{i j}=0$ for $i>j$. It is evident that the shift from the upper limit value to the midpoints for weighting each sphere size class is realized by the numerical value $1 / 2$. For example, the $(4 \times 4)$ matrix $B_{i j}$ is

$$
B_{i j}=\left(\begin{array}{cccc}
0.5 & 0.381966 & 0.208712 & 0.145898 \\
0 & 1.118034 & 0.791288 & 0.481821 \\
0 & 0 & 1.5 & 1.069506 \\
0 & 0 & 0 & 1.802776
\end{array}\right),
$$

and the sums of the matrix elements in the columns are $0.5,1.5,2.5$ and 3.5 , respectively. It should be noted that, although the formal status of the matrix in Equations 8 and 9 is the same as of that in Equations 3 and 5, its physical meaning is not. In particular, in contrast to first, the latter is not a section circle construction matrix.

One may now ask the question whether the shift of the weight factor may take another value between 0 and $1 / 2$ and whether it might go even beyond the value $1 / 2$. The answer to both questions is in principle yes. This leads us to suggest the following generalized version of the matrix $B_{i j}$ :

$$
\begin{gathered}
B_{i j}=\sqrt{(j-\alpha)^{2}-(i-1)^{2}}-\sqrt{(j-\alpha)^{2}-i^{2}} \text { for } i<j, \\
B_{i j}=\sqrt{(j-\alpha)^{2}-(i-1)^{2}}=B_{i i}= \\
=\sqrt{(i-\alpha)^{2}-(i-1)^{2}}=\sqrt{i-1+\alpha^{2}} \text { for } i=j,
\end{gathered}
$$

and $B_{i j}=0$ for $i>j$. It is evident that in the cases $\alpha=0$ and $\alpha=0.5$ the Saltykov and Cruz-Orive matrices, Equations 3 and 8, are regained, respectively. By changing the weight shift parameter $\alpha$ from 0 to values approaching unity $(\alpha \rightarrow 1)$ the whole range of possible choices is covered. Weight shift parameter values larger than 0.5 shift the weight towards the lower limit value of the size class, which should be more realistic e.g. for distributions (frequency histograms) that are steeply decreasing with increasing size. Equation 10 thus gives rise to a rather general class of transformations that include the Saltykov and Cruz-Orive transformations as special cases and provide bounds also for the Woodhead transformation, for which weighting is based on the assumption of a uniform distribution within each size class and which in practice requires the inverse of the matrix $[14,15]$

$$
\begin{aligned}
& B_{11}=\frac{1}{2} \text {, } \\
& B_{i i}=\frac{i}{2} \sqrt{i^{2}-(i-1)^{2}}-\frac{(i-1)^{2}}{2} \text {, } \\
& {\left[\ln \left\{i+\sqrt{i^{2}-(i-1)^{2}}\right\}-\ln (i-1)\right]} \\
& B_{i j}=\frac{j}{2}\left\{\sqrt{j^{2}-(i-1)^{2}}-\sqrt{j^{2}-i^{2}}\right\}-\frac{j-1}{2} . \\
& \left\{\sqrt{(j-1)^{2}-(i-1)^{2}}-\sqrt{(j-1)^{2}-i^{2}}\right\}+\frac{i^{2}}{2} \\
& {\left[\ln \left(j+\sqrt{j^{2}-i^{2}}\right)-\ln \left\{j-1+\sqrt{(j-1)^{2}-i^{2}}\right\}\right]-} \\
& -\frac{(i-1)^{2}}{2}\left[\ln \left(j+\sqrt{j^{2}-(i-1)^{2}}\right)-\right. \\
& \left.-\ln \left\{j-1+\sqrt{(j-1)^{2}-(i-1)^{2}}\right\}\right] \text { for } i<j
\end{aligned}
$$

and $B_{i j}=0$ for $i>j$. Realizing the aforementioned physical meaning, it is not very surprising that this $B_{i j}$ matrix is similar to the $B_{i j}$ matrix calculated from Equation 8 . In particular, the corresponding $(4 \times 4)$ matrix $B_{i j}$ is

$$
B_{i j}=\left(\begin{array}{cccc}
0.5 & 0.426428 & 0.212305 & 0.147019 \\
0 & 1.073572 & 0.858441 & 0.487948 \\
0 & 0 & 1.429255 & 1.152675 \\
0 & 0 & 0 & 1.712358
\end{array}\right),
$$

and the sums of the matrix elements in the columns are again $0.5,1.5,2.5$ and 3.5 , respectively.

For reasons of comparison we also provide the $(4 \times 4)$ matrix $B_{i j}$ for our generalized matrix $B_{i j}$ and a weight shift parameter $\alpha=0.999$ (this value is obviously very close to unity and thus shows the numerical behavior of our matrix when the weighting tends towards the lower limit value of the size classes). The matrix in this case is

$$
B_{i j}=\left(\begin{array}{cccc}
0.001 & 0.956267 & 0.267795 & 0.171512 \\
0 & 0.044733 & 1.669952 & 0.592078 \\
0 & 0 & 0.063253 & 2.159943 \\
0 & 0 & 0 & 0.077466
\end{array}\right) .
$$

In this case the sums of the matrix elements in the columns are $0.001,1.001,2.001$ and 3.001, respectively. 
The corresponding inverse matrices for the CruzOrive transformation, Woodhead transformation and our generalized transformation with a weight shift parameter $\alpha=0.999$ are

$$
\begin{gathered}
C_{i j}=\left(\begin{array}{cccc}
2 & -0.68328 & 0.082165 & -0.02799 \\
0 & 0.894427 & -0.47183 & 0.040867 \\
0 & 0 & 0.666667 & -0.3955 \\
0 & 0 & 0 & 0.5547
\end{array}\right), \\
C_{i j}=\left(\begin{array}{cccc}
2 & -0.79441 & 0.180055 & -0.06655 \\
0 & 0.93147 & -0.55946 & 0.111172 \\
0 & 0 & 0.699665 & -0.47098 \\
0 & 0 & 0 & 0.58399
\end{array}\right), \\
C_{i j}=\left(\begin{array}{cccc}
1000 & -21377.4 & 560151.4 & -1.5 \mathrm{E}+07 \\
0 & 22.35509 & -590.196 & 16285.23 \\
0 & 0 & 15.80941 & -440.805 \\
0 & 0 & 0 & 12.90887
\end{array}\right),
\end{gathered}
$$

respectively. If desired, all these matrices can be normalized by dividing each matrix element by the numerical value in the left top corner, i.e. $\mathrm{B}_{11}$ or $\mathrm{C}_{11}$, respectively. It is evident that the upper left corners of these matrices exhibit a checkerboard pattern of positive and negative values, in contrast to the Saltykov matrix, Equation 7, where all the diagonal elements are positive, while all the non-diagonal elements are negative. This, together with the increasing difference between neighboring matrix elements, is clearly one of the reasons why these matrices exhibit an increasingly larger tendency to statistical instability and the formation of numerical artefacts compared to the Saltykov matrix. This tendency increases as the weight shift parameter $\alpha$ increases from zero (Saltykov transformation) to unity. While for $\alpha$ values up to 0.5 (Cruz-Orive transformation) the difference between neighboring matrix elements is still relatively small and thus relatively good stability can still be expected, there is obviously a strong nonlinear increase in these differences for $\alpha$ values exceeding a critical range. In the next section we will investigate this transition from the "safe" range of $\alpha$ values to the critical range beyond which the transformation leads to completely wrong results.

We would like to emphasize that histograms with only 4 size classes and thus also $(4 \times 4)$ matrices are of course not very useful in practice. Here they serve only for the purpose of illustration and mutual comparison. Using Equations 3, 8, 10 and 11 it is straightforward to calculate the corresponding matrices for an arbitrary number of size classes in any common table calculator (e.g. MS Excel ${ }^{\circledR}$ ) and perform the inversion of these matrices whenever desired. Most common are histograms with up to 15 size classes. The $(4 \times 4)$ matrices given in this paper represent the upper left corner of these matrices and may thus serve as a short check that the calculation formulae have been correctly inserted.

Finally we note in passing that, tacitly correcting a few misprints in Wicksell's paper [1], the $B_{i j}$ matrix leading to the Wicksell transformation [3] is

$$
\begin{gathered}
B_{i j}=\sqrt{j^{2}-\left(i-\frac{1}{2}\right)^{2}}-\sqrt{j^{2}-\left(i+\frac{1}{2}\right)^{2}} \text { for } i<j, \\
B_{i j}=\sqrt{j^{2}-\left(i-\frac{1}{2}\right)^{2}}=B_{i i}= \\
=\sqrt{i^{2}-\left(i-\frac{1}{2}\right)^{2}}=\sqrt{i-\frac{1}{4}} \text { for } i=j,
\end{gathered}
$$

and $B_{i j}=0$ for $i>j$. Due to Wicksell's unusual representation of the histogram, with the first full interval starting at the midpoint of the first size class instead of zero, it is necessary to add ad hoc definitions for the values in the first line of this matrix. These are $B_{00}=1 / 4$ and

$$
B_{0 j}=j-\sqrt{j^{2}-\frac{1}{4}}
$$

It is evident that the numerical value $1 / 2$ is responsible for shifting the size class position by half an interval width. Thus, introducing a second parameter (position shift parameter) $\beta$ it would be straightforward to extend our general matrix formula to include also Wicksell's matrix. A basic condition that must be fulfilled for this matrix to be defined is $\alpha+\beta<1$. However, although in principle also this parameter can be changed from 0 to 1 , it is useful to adhere to the convention to fix this parameter always to a value of 1 , which is tantamount with the commonly used standard representation of histograms today. Therefore, instead of giving here this purely formal extension, we give - for reasons of completeness - the $(4 \times 4)$ matrices $B_{i j}$ and $C_{i j}$ of the Wicksell transformation, which are

$$
B_{i j}=\left(\begin{array}{cccc}
0.25 & 0.133975 & 0.063508 & 0.04196 \\
0 & 0.866025 & 0.613616 & 0.359964 \\
0 & 0 & 1.322876 & 0.939764 \\
0 & 0 & 0 & 1.658312
\end{array}\right) .
$$

(column sums $0.25,1,2$ and 3, respectively) and

$$
C_{i j}=\left(\begin{array}{cccc}
4 & -0.6188 & 0.095 & -0.02073 \\
0 & 1.154701 & -0.53561 & 0.052882 \\
0 & 0 & 0.755929 & -0.42838 \\
0 & 0 & 0 & 0.603023
\end{array}\right),
$$

The Wicksell transformation can e.g. be used when the data are available in the form of a histogram that starts from the midpoint of the first interval; in this case (where the first line and the first column should 
be eliminated) it replaces the Saltykov transformation. The Wicksell transformation should generally not be used with histograms in the standard representation, i.e. starting from zero. A Wicksell-type analogue of the Cruz-Orive transformation is not defined, because when $\alpha=\beta=0.5$ the aforementioned condition $\alpha+\beta<1$ is not fulfilled.

\section{PERFORMANCE OF OUR GENERALIZED TRANSFORMATION MATRIX}

In this section we investigate the performance of our generalized transformation matrix with different values of the weight shift parameter $\alpha$ from 0 to 0.999 when applied to different model distributions (paradigmatic examples): Monodisperse, bidisperse, linear (triangular) increasing, uniform (rectangular), linear (triangular) decreasing and Rayleigh. In a first step a section circle diameter distribution has been constructed from a given sphere diameter distribution using Equation 1 and the section circle construction matrix defined via Equation 3. Then, in a second step, transformation matrices with a different value of the weight shift parameter $\alpha$ are applied in an attempt to reestablish (reconstruct) the original sphere diameter distribution. The number of size classes (bins) used in all examples is 15, but in some of the examples several bins are empty.

The three-dimensional figures (labeled "b") show the attempts of reconstructing the sphere diameter distribution (shown in the front $x-y$ plane) in dependence of the weight shift parameter (in $z$ direction). A weight shift parameter value of $\alpha=0$ corresponds to the Saltykov transformation (i.e. Equation 6 together with the inverse of Equation 3), which exactly restores the original sphere diameter distribution, because it is exactly the inverse of Equation 2 combined with Equation 3, by which the section circle distribution has been constructed. A weight shift parameter value of $\alpha=0.5$ corresponds to the Cruz-Orive transformation (i.e. Equation 6 together with the inverse of Equation 8). In all these figures the $x-y$ planes show discrete cumulative distribution "curves", representing the corresponding histograms via the upper limit values of their size classes. The two-dimensional graphs (labeled "a") show the corresponding discrete frequency distribution "curves" (also representing histograms via the upper limit values of their size classes).

Figures 1a (frequencies) and 1b (cumulative numbers) refers to a monodisperse sphere size distribution consisting of a single size class (all spheres are assumed to have a size of $7.5 \mu \mathrm{m}$, i.e. all lie in the interval $7-8 \mu \mathrm{m}$ and their arithmetic mean diameter is $7.5 \mu \mathrm{m})$. It is evident that the reconstruction fails completely, i.e. shifts the section circle diameter distribution into the wrong direction, when the weight shift parameter value is $\alpha=$ $=0.8$ or higher. In this case the reconstruction process goes into the wrong direction and - apart from exhibiting large oscillations (numerical instability), overshoots to positive and even negative unphysical values - shifts the distribution histogram towards zero, i.e. the original sphere diameter distribution cannot be reconstructed at all. When the weight shift parameter value is $\alpha=0.7$ and lower, the reconstruction shows at least a qualitative similarity with the original sphere size distribution, although the oscillations are still large (numerical instability) and both positive and negative overshoots still occur. The latter are characteristic also for the Cruz-Orive transformation $(\alpha=0.5)$ and are completely eliminated only for the Saltykov transformation $(\alpha=0)$.

Figures $2 \mathrm{a}$ and $2 \mathrm{~b}$ refer to a bidisperse sphere size distribution consisting of two size classes (one class with $2.5 \mu \mathrm{m}$ diameter spheres and one with $11.5 \mu \mathrm{m}$ spheres,

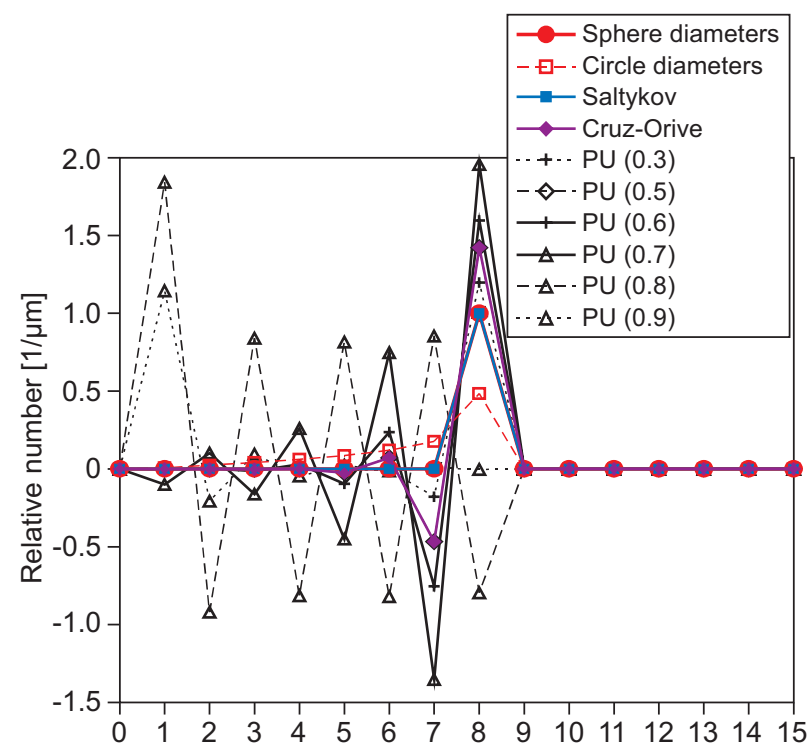

a) frequency histograms

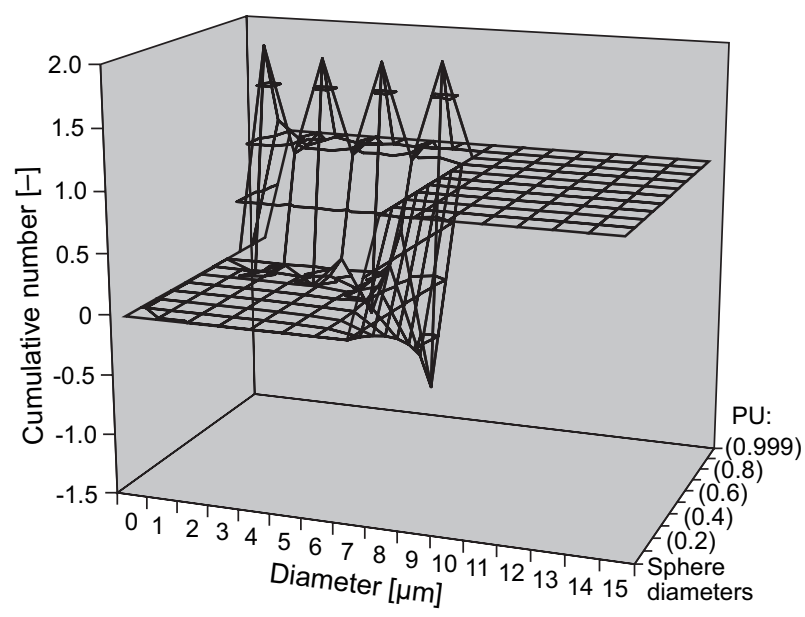

b) cumulative histograms

Figure 1. Monodisperse sphere diameter distribution (7-8 $\mu \mathrm{m}$, arithmetic mean $7.5 \mu \mathrm{m}$ ) and its reconstruction by the generalized transformation with weight shift parameter values between 0 and 0.9 ; a) frequency histograms, b) cumulative histograms. 
arithmetic mean $7 \mu \mathrm{m})$. Also in this case the reconstruction fails completely or is very bad when $\alpha>0.7$. For values $\alpha=0.7$ and lower, the reconstruction shows all qualitative features of the Cruz-Orive transformation $(\alpha=0.5)$, including numerical artefacts, which are eliminated only for the Saltykov transformation $(\alpha=0)$.

Figures $3 \mathrm{a}, 3 \mathrm{~b}, 4 \mathrm{a}$ and $4 \mathrm{~b}$ all refer to increasing linear (triangular saw-tooth) distributions, Figures $3 \mathrm{a}$ and $3 \mathrm{~b}$ starting with the size class $1-2 \mu \mathrm{m}$ and ending with the size class $10-11 \mu \mathrm{m}$ (arithmetic mean $7.5 \mu \mathrm{m}$ ), Figures $4 \mathrm{a}$ and $4 \mathrm{~b}$ starting with the size class $2-3 \mu \mathrm{m}$ and ending with the size class $11-12 \mu \mathrm{m}$ (arithmetic mean $8.5 \mu \mathrm{m}$ ). In both cases the reconstruction fails completely or is very bad when $\alpha \geq 0.8$ and relatively good when

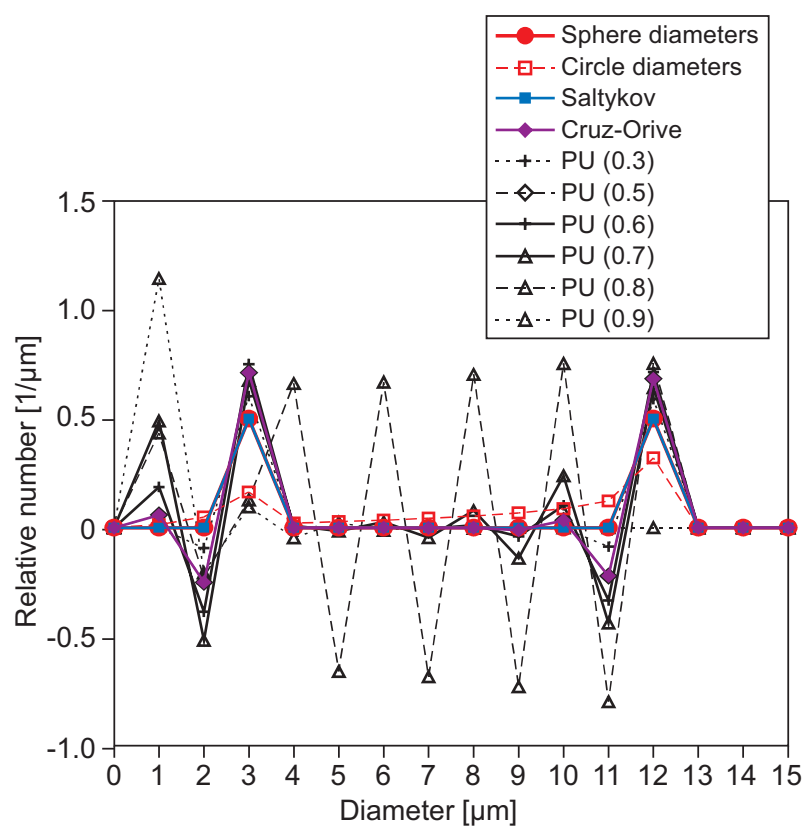

a) frequency histograms

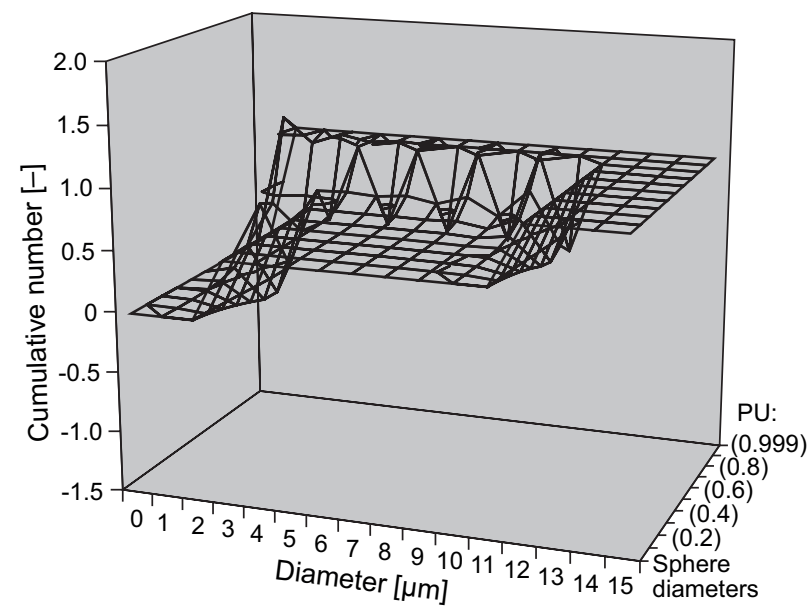

b) cumulative histograms

Figure 2. Bidisperse sphere diameter distribution $(2-3 \mu \mathrm{m}$ and 11-12 $\mu \mathrm{m}$, arithmetic mean $7 \mu \mathrm{m}$ ) and its reconstruction by the generalized transformation with weight shift parameter values between 0 and 0.9 ; a) frequency histograms, b) cumulative histograms. $\alpha \leq 0.7$. However, in the first case (Figures $3 a$ and $3 b$ ) the cumulative curves approach the Cruz-Orive and Saltykov transformation results in a monotonous way from above, whereas in the second case the transformation with $\alpha=0.8$ exhibits unphysical overshoots to negative numbers, obviously as a consequence of the numerical instability of transformations with such high weight shift parameter values, and for $\alpha \geq 0.8$ approach the Cruz-Orive and Saltykov transformation results in a monotonous way from below.

In the case of decreasing linear (triangular sawtooth) distributions starting with the same size class ranges as above (arithmetic means $4.5 \mu \mathrm{m}$ and $5.5 \mu \mathrm{m}$, respectively, see Figures 5a, 5b, 6a and $6 \mathrm{~b}$ ), the situation is similar, but with the two cases reversed: the reconstruction fails completely or is very bad when $\alpha \geq 0.8$

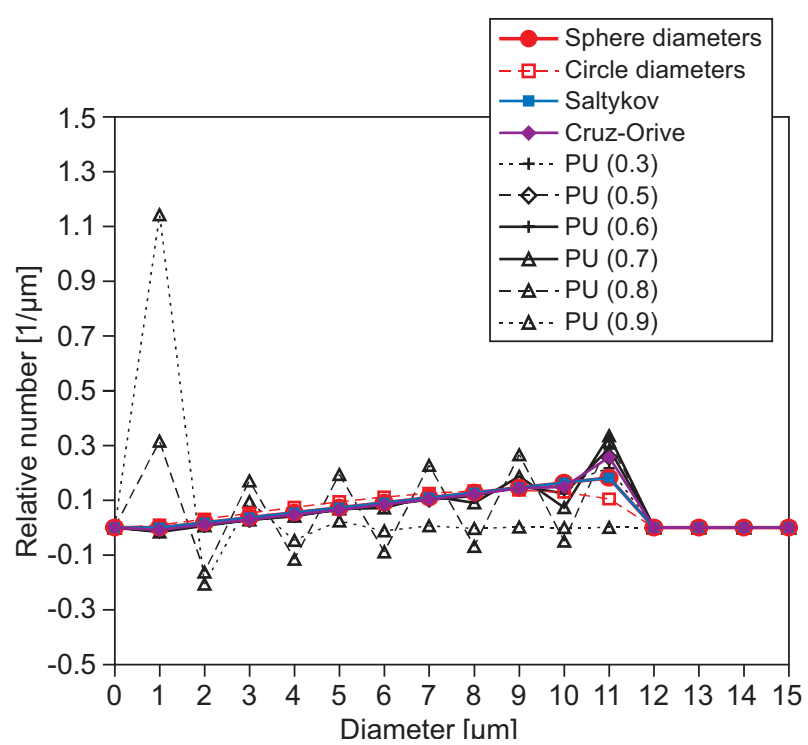

a) frequency histograms

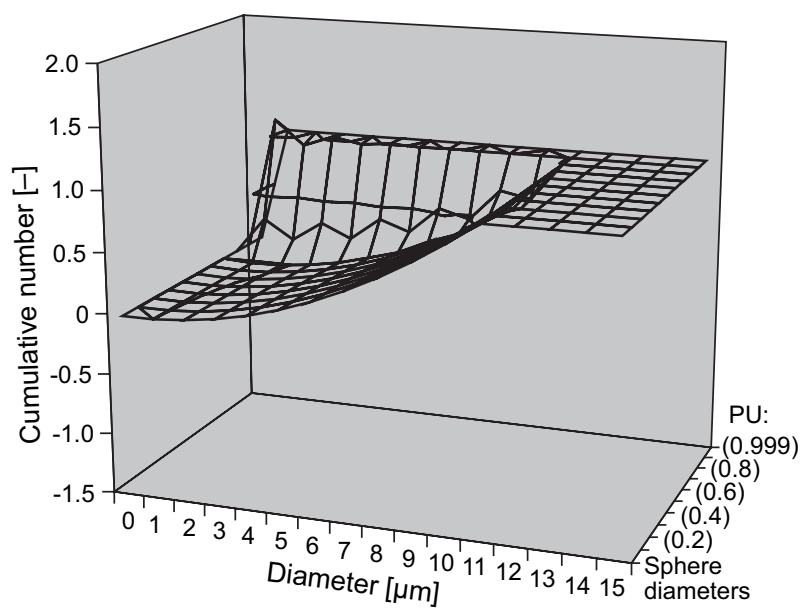

b) cumulative histograms

Figure 3. Increasing linear (triangular saw-tooth) sphere diameter distribution from interval $1-2 \mu \mathrm{m}$ to interval $10-11 \mu \mathrm{m}$, arithmetic mean $7.5 \mu \mathrm{m}$ ) and its reconstruction by the generalized transformation with weight shift parameter values between 0 and 0.9 ; a) frequency histograms, b) cumulative histograms. 
and relatively good when $\alpha \leq 0.7$. However, in the first case (Figures $5 \mathrm{a}$ and $5 \mathrm{~b}$ ) the cumulative curves approach the Cruz-Orive and Saltykov transformation results in a monotonous way from below, exhibiting unphysical overshoots to negative numbers, whereas in the second case the Cruz-Orive and Saltykov transformation results are approached in a monotonous way from above. In the case of decreasing linear distributions, in contrast to increasing linear distributions, negative overshoots are exhibited also by the Cruz-Orive transformation. Only the Saltykov transformation is free of these effects in this case. The example of two similar distributions differing only by their starting point, clearly shows that the beha-

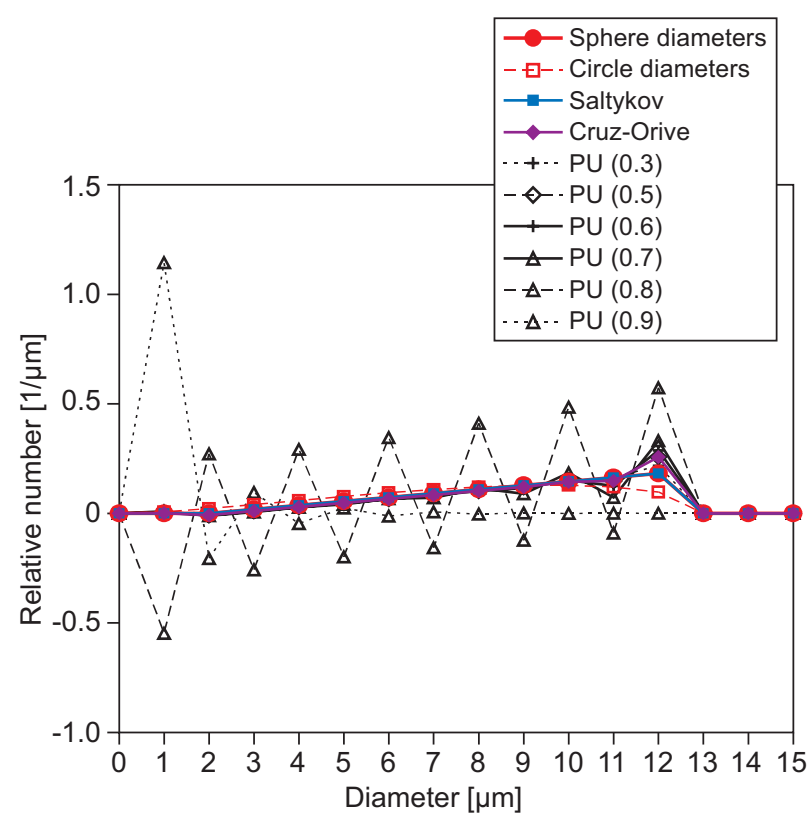

a) frequency histograms

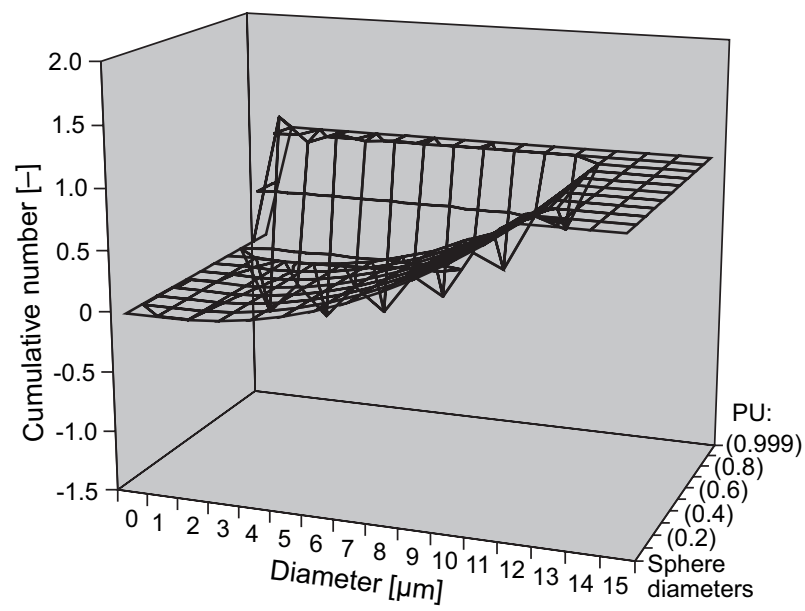

b) cumulative histograms

Figure 4. Increasing linear (triangular saw-tooth) sphere diameter distribution from interval 2-3 $\mu \mathrm{m}$ to interval $11-12 \mu \mathrm{m}$, arithmetic mean $8.5 \mu \mathrm{m}$ ) and its reconstruction by the generalized transformation with weight shift parameter values between 0 and 0.9 ; a) frequency histograms, b) cumulative histograms. vior of numerically unstable transformations cannot be easily predicted and that general statements about their behavior should be made with utmost caution.

Figures $7 \mathrm{a}$ and $7 \mathrm{~b}$ refer to a uniform (rectangular) sphere size distribution that exhibits a constant frequency between the size classes 2 - $3 \mu \mathrm{m}$ and 11 - $12 \mu \mathrm{m}$ (arithmetic mean $7 \mu \mathrm{m}$ ), see Figure $7 \mathrm{a}$. The corresponding cumulative numbers exhibit a linear increase, see Figure 7b. Curiously, in this case the reconstruction fails completely or is very bad only for transformations with $\alpha \geq 0.9$, while already transformations with $\alpha \leq 0.8$ are numerically very stable and approach the Cruz-Orive and Saltykov transformation results rather closely. The same conclusion holds for the Rayleigh distribution example shown in Figures 8a and 8b (arithmetic mean 6.108 $\mu \mathrm{m}$ ).

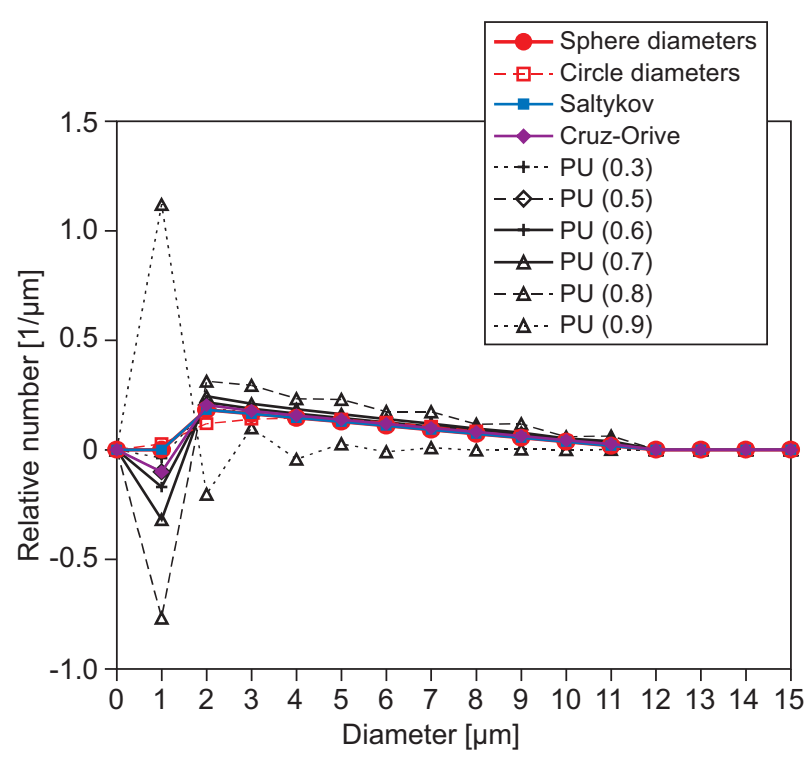

a) frequency histograms

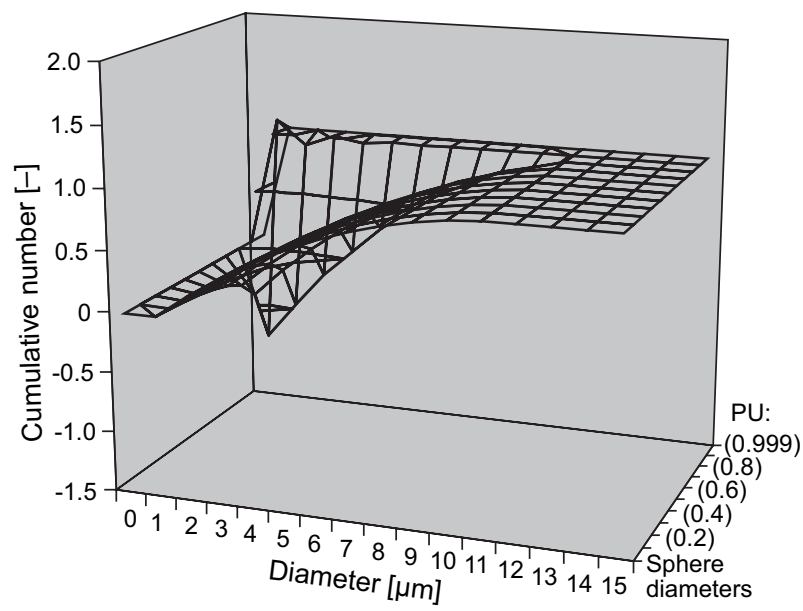

b) cumulative histograms

Figure 5. Decreasing linear (triangular saw-tooth) sphere diameter distribution from interval $1-2 \mu \mathrm{m}$ to interval $10-11 \mu \mathrm{m}$, arithmetic mean $4.5 \mu \mathrm{m}$ ) and its reconstruction by the generalized transformation with weight shift parameter values between 0 and 0.9 ; a) frequency histograms, b) cumulative histograms. 


\section{SUMMARY AND CONCLUSIONS}

In this paper a generalized formulation of transformation matrices has been proposed for the reconstruction of sphere diameter distributions from their section circle diameter distributions. This generalized formulation is based on a weight shift parameter $\alpha$ that can be adjusted from 0 to 1 . It includes the well-konwn Saltykov and Cruz-Orive transformations as special cases (for parameter values of $\alpha=0$ and $\alpha=0.5$, respectively). The transformation exploits the fact that larger spheres embedded in a microstructure have a higher probability to be cut by a random plane (planar section) than smaller spheres, but allows for the possibility that the center of inertia of the size class is not its upper limit

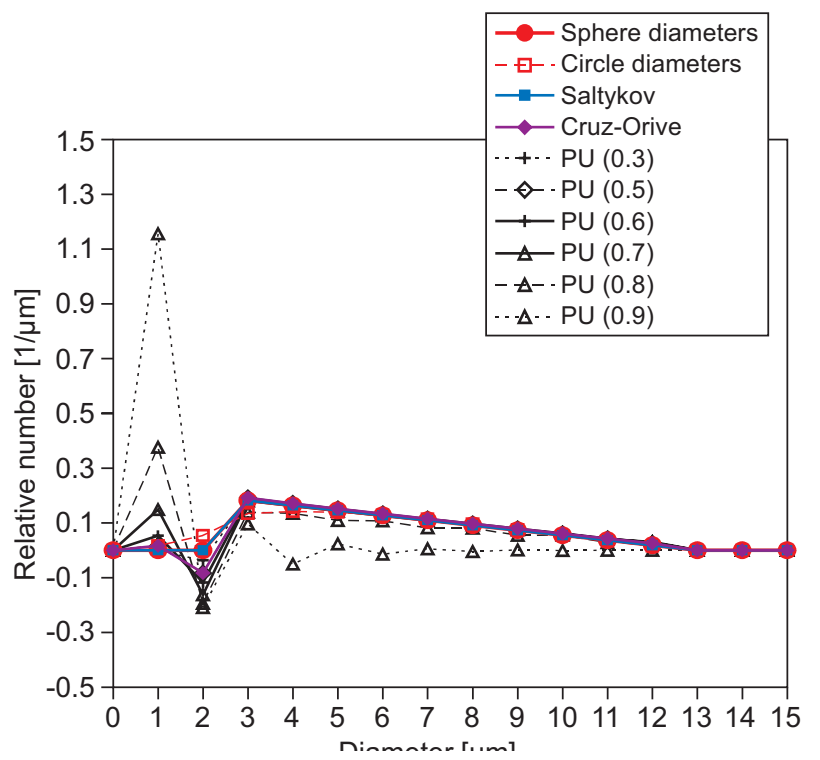

a) frequency histograms

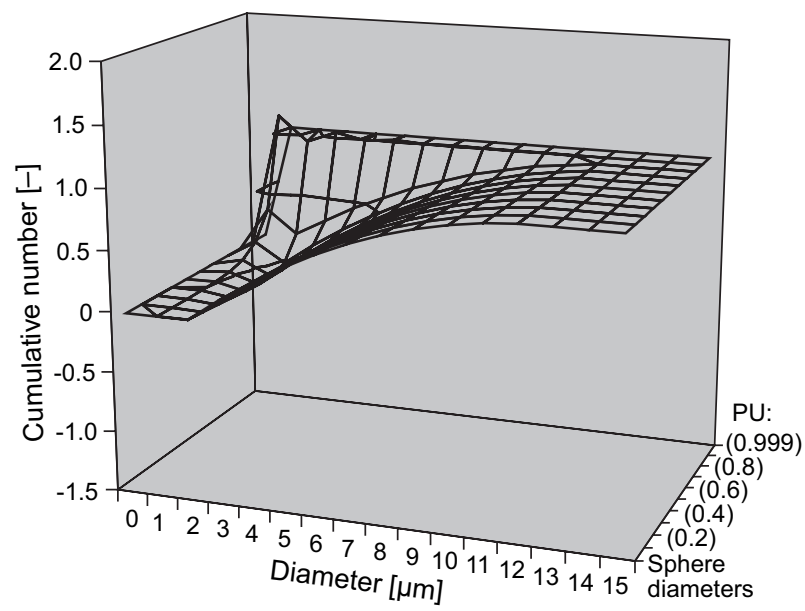

b) cumulative histograms

Figure 6. Decreasing linear (triangular saw-tooth) sphere diameter distribution from interval 2-3 $\mu \mathrm{m}$ to interval $11-12 \mu \mathrm{m}$, arithmetic mean $5.5 \mu \mathrm{m}$ ) and its reconstruction by the generalized transformation with weight shift parameter values between 0 and 0.9 ; a) frequency histograms, b) cumulative histograms. value (Saltykov) or its midpoint (Cruz-Orive), but can be closer to the lower limit value $\alpha \rightarrow 1$. It seems plausible that this possibility should not be excluded a priori, because it could be more adequate e.g. for the decreasing branches of frequency curves or histograms. Moreover, when both extreme cases $(\alpha=0$ and $\alpha \rightarrow 1)$ are considered it is clear that not only the Cruz-Orive transformation, but also the Woodhead transformation, which assumes a uniform distribution inside each sphere size class, should be somewhere inside the two extreme cases. However, our investigation of paradiagmatic examples has shown that our generalized transformation is numerically highly unstable, i.e. will introduce numerical artefacts (oscillations or even unphysical negative sphere frequencies) into the reconstruction,

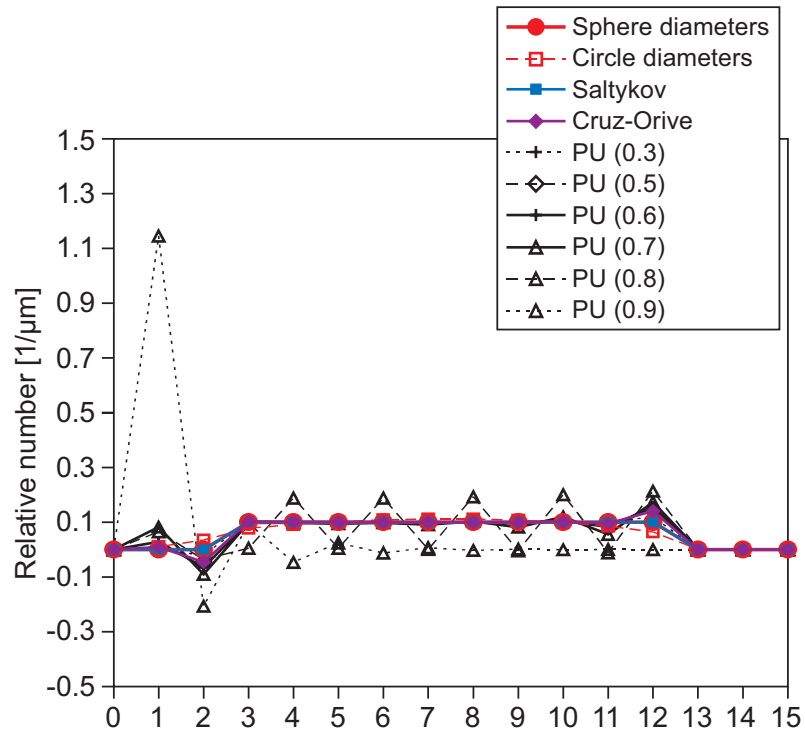

a) frequency histograms

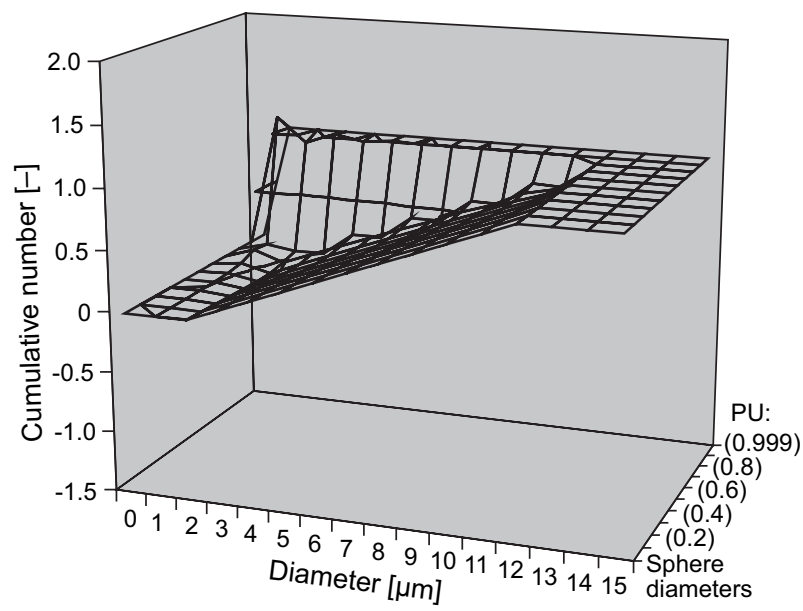

b) cumulative histograms

Figure 7. Uniform (rectangular) sphere diameter distribution from interval 2-3 $\mu \mathrm{m}$ to interval $11-12 \mu \mathrm{m}$, arithmetic mean $7 \mu \mathrm{m})$ and its reconstruction by the generalized transformation with weight shift parameter values between 0 and 0.9 ; a) frequency histograms, b) cumulative histograms. 
and can lead to completely wrong results when a critical value of the parameter (usually in the range $0.7-0.9$, depending on the type of distribution) is exceeded. It has been shown that the mentioned numerical instability is an intrinsic feature of these transformations that depends not only on the weight shift parameter value and is affected both by the type and the position of the distribution. It occurs in a natural way also for the CruzOrive and other transformations with finite weight shift parameter values and is not just caused by inadequate input data (e.g. as a consequence of an insufficient number of objects counted), as commonly assumed. Finally it has been shown that an even more general class of transformation matrices can be defined that includes, in addition to the aforementioned transformations, also the Wicksell transformation.

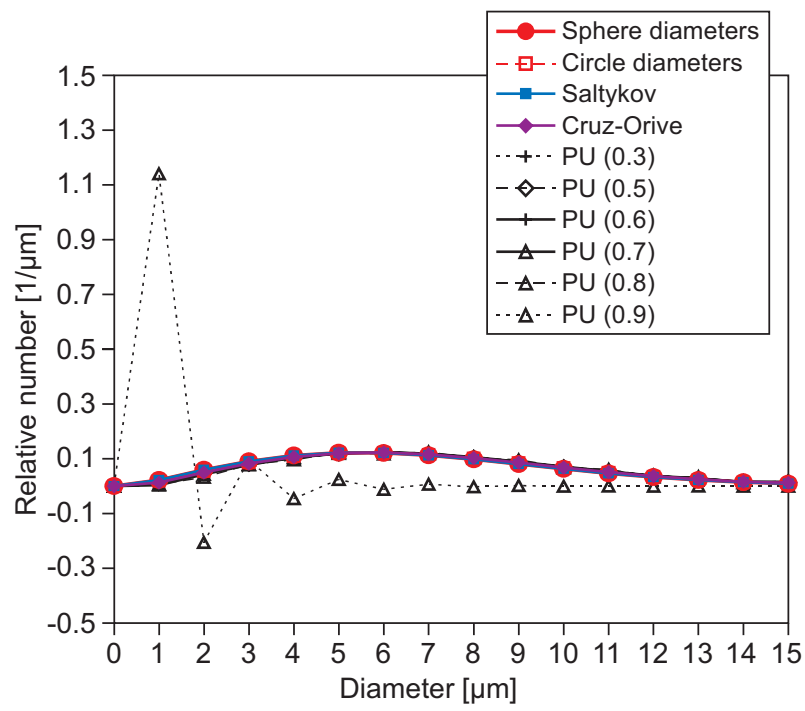

a) frequency histograms

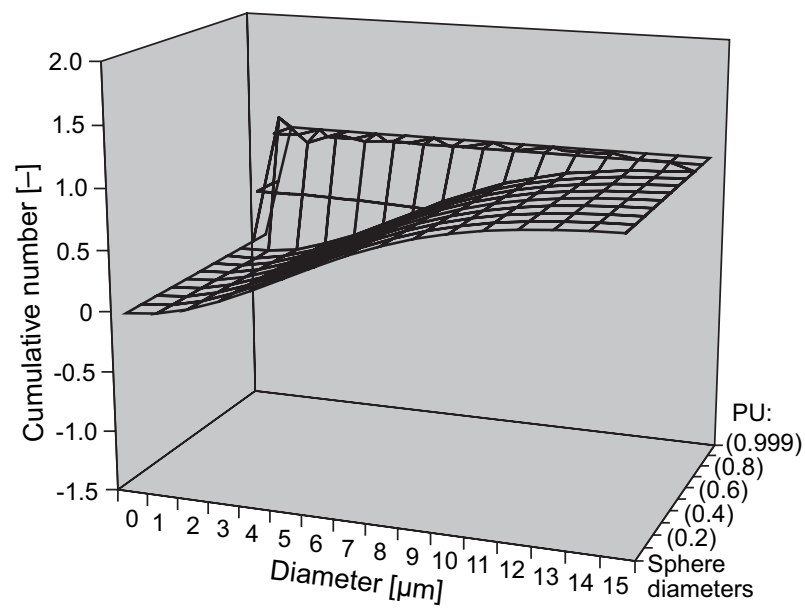

b) cumulative histograms

Figure 8. Rayleigh distribution of sphere diameters with (arithmetic mean theoretical $6.15452 \mu \mathrm{m}$, arithmetic mean from histogram $6.10845 \mu \mathrm{m}$ ) and its reconstruction by the generalized transformation with weight shift parameter values between 0 and 0.9 ; a) frequency histograms, b) cumulative histograms.
Acknowledgement

This work is part of the project "Preparation and characterization of oxide and silicate ceramics with controlled microstructure and modeling of microstructure-property relations" (GA15-18513S), supported by the Czech Science Foundation (GAČR).

\section{REFERENCES}

1. Wicksell S. D. (1925): The corpuscle problem - A mathematical study of a biometric problem. Biometrika, 17, 84-99. doi:10.1023/A:1020982025786

2. Wicksell S. D. (1926): The corpuscle problem - Second memoir: case of ellipsoidal corpuscles. Biometrika, 18, 151-172. doi:10.2307/2332500

3. Weibel E. R. (1980): Stereological Methods (Volume 2 Theoretical Foundations). Academic Press.

4. Škrášek J., Tichý Z. (1990): Základy aplikované matematiky III (Fundamentals of Applied Mathematics, in Czech). SNTL / Státní nakladatelství technické literatury.

5. Scheil E. (1931): Die Berechnung der Anzahl der Grössenverteilung kugelförmiger Kristalle in undurchsichtigen Körpern mit Hilfe der durch einen ebenen Schnitt erhaltenen Schnittkreise. Zeitschrift für Anorganische und Allgemeine Chemie, 27, 259-264. doi:10.1002/zaac.19312010123

6. Schwartz H. A. (1934): The metallographic determination of the size distribution of temper carbon nodules. Metals and Alloys, 5, 139-140.

7. Saltykov S. A. (1958). Stereometric Metallography. $2^{\text {nd }}$ ed. (in Russian). State Publishing House for Metals and Sciences.

8. Saltykov S. A. (1974). Stereometrische Metallographie. VEB Deutscher Verlag für Grundstoffindustrie.

9. Saltykov S. A. (1970). Stereometric Metallography. $3^{\text {rd }}$ ed. (in Russian). State Publishing House for Metals and Sciences.

10. Saltykov S. A. (1968). Stereometrická metalografie. SNTL (Státní nakladatelství technické literatury).

11. Cruz-Orive L. M. (1976): Particle size-shape distributions: the general spheroid problem - I. Mathematical model. Journal of Microscopy, 107, 235-253. doi:10.1111/ j.13652818.1976.tb02446.x

12. Cruz-Orive L. M. (1978): Particle size-shape distributions: the general spheroid problem - II. Stochastic model and practical guide. Journal of Microscopy, 112, 153-167. doi:10.1111/j.1365-2818.1978.tb01162.x

13. Goldsmith P. L. (1967): The calculation of true particle size distributions from the sizes observed in a thin slice. British Journal of Applied Physics, 18, 813-830. doi:10.1088/0508$3443 / 18 / 6 / 317$

14. Higginson R. L., Sellars C. M. (2003). Worked Examples in Quantitative Metallography. Maney.

15. Blödner R., Mühlig P., Nagel W. (1984): The comparison by simulation of solutions of Wicksell's corpuscle problem. Journal of Microscopy, 135, 61-74. doi:10.1111/ j.13652818.1984.tb04649.x

16. Underwood E. E. (1970). Quantitative Stereology. AddisonWesley.

17. Lewis H. D., Walthers K. L., Johnson K. A. (1973): Particle size distributions by area analysis - Modifications of the 
Saltykov method. Metallography, 6, 93-101. doi:10.1016/ 0026-0800(73)90001-3

18. Uhlirová T., Pabst W., Gregorová E., Hostaša J. (2016): Stereology of dense polycrystalline materials - from interface density and mean curvature integral density to Rayleigh distributions of grains sizes. Journal of the European Ceramic Society, 36, 2319-2328. doi:10.1016/j. jeurceramsoc.2015.09.028

19. Anderssen R. S., Jakeman A. J. (1975): Abel type integral equations in stereology - I. General discussion. Journal of Microscopy, 105, 121-133. doi:10.1111/j.1365-2818.1975. tb04045.x

20. Anderssen R. S., Jakeman A. J. (1975): Abel type integral equations in stereology - II. Computational methods of solution and the random spheres approximation, Journal of Microscopy, 105, 135-153. doi:10.1111/j.1365-2818.1975. tb04046.x

21. Lord K. A. (2013). Methods for obtaining true particle size distributions from cross section measurements. Dissertation (Paper 13221). Iowa State University.
22. Gerlach W., Ohser J. (1986): On the accuracy of numerical solution for some stereological problems such as the Wicksell corpuscle problem, Biometrical Journal, 28, 881-887. doi:10.1002/bimj.4710280719

23. Stoyan D., Kendall W. S., Mecke J. (1995). Stochastic Geometry and its Applications. $2^{\text {nd }}$ ed. John Wiley \& Sons.

24. Uhlirova T., Pabst W. Reconsideration of Wicksell's random section problem for spherical inclusions and comparison of the performance of transformation matrices on model distributions. in preparation

25. Rysz J., Wiencek K. (1980): Stereology of spherical carbide particles in steels. Archiv Nauki o Materialach, 1, 151-168.

26. Drees H., Reiss R. D. (1992): Tail behavior in Wicksell's corpuscle problem, pp. 205-220 in Galambos J., Katai I. (eds.): Probability and Applications. Kluwer. 